\title{
Universal Relaxational Dynamics of Gapped One- Dimensional Models in the Quantum Sine-Gordon Universality Class
}

\section{Citation}

Damle, Kedar, and Subir Sachdev. 2005. "Universal Relaxational Dynamics of Gapped OneDimensional Models in the Quantum Sine-Gordon Universality Class." Physical Review Letters 95 (18). https://doi.org/10.1103/physrevlett.95.187201.

\section{Permanent link}

http://nrs.harvard.edu/urn-3:HUL.InstRepos:41417301

\section{Terms of Use}

This article was downloaded from Harvard University's DASH repository, and is made available under the terms and conditions applicable to Other Posted Material, as set forth at http:// nrs.harvard.edu/urn-3:HUL.InstRepos:dash.current.terms-of-use\#LAA

\section{Share Your Story}

The Harvard community has made this article openly available. Please share how this access benefits you. Submit a story. 


\title{
Universal relaxational dynamics of gapped one dimensional models in the quantum sine-Gordon universality class
}

\author{
Kedar Damle ${ }^{1}$ and Subir Sachdev ${ }^{2}$ \\ ${ }^{1}$ Department of Theoretical Physics, Tata Institute of Fundamental Research, Mumbai 400005, India \\ ${ }^{2}$ Department of Physics, Harvard University, Cambridge, MA 02138
}

(Dated: September 14, 2017)

\begin{abstract}
A semiclassical approach to the low-temperature real time dynamics of generic one-dimensional, gapped models in the sine-Gordon model universality class is developed. Asymptotically exact universal results for correlation functions are obtained in the temperature regime $T \ll \Delta$, where $\Delta$ is the energy gap.

PACS numbers: 75.10.Jm 05.30.Jp 71.27.+a
\end{abstract}

Real time, non-zero temperature $(T)$, correlation functions of quantum many body systems are directly related to many experimentally measurable quantities. For strongly interacting systems, there are few quantitative results on the relaxation and transport processes that are believed to occur at long times at any $T \neq 0$. Standard perturbative methods, as well as many numerical approaches, work best in imaginary time, but the analytic continuation of such imaginary time results to real time is most dangerous, and often fails, in the low frequency limit. Among the few exact results available are those describing relaxational behaviour of an order parameter at conformally invariant critical points (obtained by exploiting the conformal invariance of the system), or by semiclassical methods deep in an ordered state [1], both in dimension $d=1$. In an important recent development, Altshuler and Tsvelik 2] (AT) obtained real time order parameter correlations for the integrable $d=1$ quantum Ising model by the form-factor expansion, and their results were in precise agreement with the semiclassical predictions [1].

AT also argued that their results could be extended to other gapped, integrable systems, and in particular, to the sine-Gordon model; some results for another massive integrable model (the $\mathrm{O}(3)$ non-linear sigma model) had also been obtained earlier by Fujimoto $[\underline{3}$ and Konik [4]. In this paper, we obtain dynamic non-zero $T$ correlators of gapped one dimensional models in the universality class of the $d=1$ quantum sine-Gordon field theory using a semiclassical method [1]: our results are expected to apply generic, non-integrable models 5 . Interestingly, as we describe in detail below, our semiclassical results are qualitatively different from those of AT. One likely origin of this difference is that the long time transport and spectral [6] properties of integrable models, with infinitely many conserved charges [], are genuinely distinct from those of more generic models; the latter have only a small number of conserved charges and are described by the semiclassical theory. The generic quantum Ising model has only a single conserved charge (the energy density) and was possibly too simple a model to expose the above distinction. At the end of this paper, we will also mention other possible sources of the difference between our sine-Gordon results and those of AT and others [3, 4].

The imaginary time $(\tau)$ action of the sine-Gordon model is

$$
\mathcal{A}=\frac{c}{16 \pi} \int_{0}^{1 / T} d \tau d x\left[\left(\partial_{x} \Phi\right)^{2}+\frac{1}{c^{2}}\left(\partial_{\tau} \Phi\right)^{2}-g^{2} \cos (\gamma \Phi)\right]
$$

where $\Phi$ is the sine-Gordon field, and $c$ has dimensions of velocity. It is assumed that the full integrability of the model is broken either by additional terms not shown above, or by an ultraviolet cutoff that does not preserve integrability. Such perturbations are always present in any experimentally relevant realization (see Ref. 7 for examples of experimental applications), but will be left implicit in our discussion below; we will thus freely refer to the sine-Gordon model when we mean generic models in this universality class. In the integrable sine-Gordon theory, $g$ is relevant for $\gamma<1$, giving rise to a gapped phase with particle-like excitations. For $1 / \sqrt{2}<\gamma<1$, the interactions between these particles are purely repulsive and there are no bound states. It is expected that a similar 'purely repulsive' regime exists without full integrability.

Our focus here is on the space and time dependent correlator of $e^{i \eta \Phi}$ defined in the usual way for arbitrary $\eta: C_{\Phi}(x, t) \equiv\left\langle e^{i \eta \Phi(x, t)} e^{-i \eta \Phi(0,0)}\right\rangle$, where $\Phi(x, t) \equiv$ $e^{i \hat{\mathcal{H}} t} \Phi(x) e^{-i \hat{\mathcal{H}} t}, \hat{\mathcal{H}}$ is the Hamiltonian of the system, and the angular brackets indicate average over the equilibrium density matrix of the system. Below, we shall obtain the space-time dependent $C(x, t)$ for temperatures $T \ll \Delta$ ( $\Delta$ is the gap) in the purely repulsive regime of the gapped phase mentioned above. Our answer will be a universal scaling function of $x, t, \Delta$ and $c$, a velocity that enters the dispersion relation of the solitonic excitations.

There are three key observations that allow our exact computation for $T \ll \Delta$. The first is that as there is an excitation gap, and the lowest lying excitations above the gap are 'charged' particles (with charges \pm 1 ) that represent solitons and anti-solitons in the sine-Gordon 
field. For $T \ll \Delta$, the particle dispersion $\epsilon(p)$ may be set equal to its universal low momentum form $\epsilon(p)=\Delta+$ $p^{2} c^{2} / 2 \Delta$, and they may be treated semiclassicaly using Maxwell-Boltzmann statistics with a velocity probability density $P(v) \sim e^{-\Delta v^{2} / 2 c^{2} T}$. Indeed, their density

$$
\rho=2 e^{-\Delta / T} \int \frac{d p}{2 \pi} e^{-p^{2} c^{2} /(2 \Delta T)},
$$

while their r.m.s. velocity $v$ is $v_{T}=c(T / \Delta)^{1 / 2}$. The mean inter-particle spacing $\sim e^{+\Delta / T}$ is thus much larger than the thermal de-Broglie wavelength $\sim c /(\Delta T)^{-1 / 2}$, and the particles behave semiclassically $[1]$.

The second observation is that collisions between these particles are described by their two-particle $S$-matrix, and only a simple universal low-velocity limit of this $S$ matrix is needed in the low $T$ limit since the particle 'rapidity' $\sim v_{T} / c \ll 1$. In this limit, in the purely repulsive regime of the gapped phase, the $S$-matrix for the process in Fig [1: is generically []

$$
\mathcal{S}_{m_{1}^{\prime}, m_{2}^{\prime}}^{m_{1} m_{2}}=(-1) \delta_{m_{1} m_{2}^{\prime}} \delta_{m_{2} m_{1}^{\prime}} .
$$

In other words, the excitations behave like impenetrable particles which preserve their charge in a collision. Energy and momentum conservation in $d=1$ require that these particles simply exchange momenta across a collision (Fig 15). The (-1) factor in Eq. (2) can be interpreted as the phase-shift of repulsive scattering between slowly moving bosons in $d=1$. Indeed, the simple generic form of Eq. (2) is due to the slow motion of the particles [5], and is not a special feature of relativistic continuum theory. It is also worth noting that at special free fermion points in the gapped phase (when a mapping to non-interacting fermions exists), the repulsive interaction between the solitonic particles goes to zero and the $S$-matrix is then perfectly transmitting i.e. it equals $(-1) \delta_{m_{1} m_{1}^{\prime}} \delta_{m_{2} m_{2}^{\prime}}$ (for generic, non-integrable models, accessing this free fermion behavior requires tuning an infinite number of couplings, and so this point is not physically relevant). The dynamics in this special case is expected to be very different from the generic behavior, and will be discussed below as a prelude to our results in the generic case.

Finally, we note that in the semiclassical limit at low temperatures, for most of the time, the sine-Gordon field $\Phi$ takes one of the values

$$
\Phi=\frac{2 \pi n}{\gamma}
$$

where $n$ is an integer which increases (decreases) upon crossing in the positive $x$ direction a soliton (anti-soliton) trajectory. A sample set of soliton trajectories in spacetime are shown in Fig 1 along with the associated values of $n$. Notice the difference in the $n$ values between the transmitting soliton free-fermion case, and the generic reflecting soliton case described by Eq. (2).
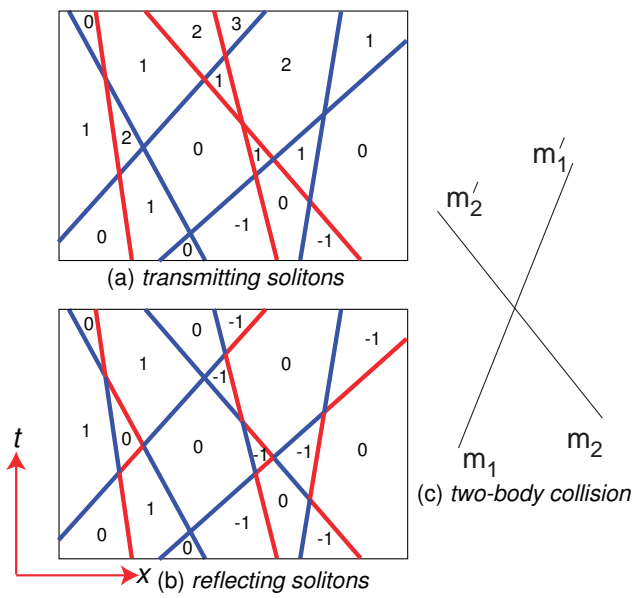

FIG. 1: A typical set of particle trajectories contributing to $C_{\Phi}(x, t)$. Each trajectory represents paths moving both forward and backward in time. The charge moving on a trajectory is indicated by different colors (color online): Red corresponds to solitons $(m=+1)$ and blue to anti-solitons $(m=-1)$. The numbers in the domains are the local values of the integer $n$ in Eq. (3)

We now present our final results for $C_{\Phi}(x, t)$, deferring their derivation till later. For the transmitting soliton case in Fig 1, an elementary computation shows that

$$
C_{\Phi}(x, t)=\mathcal{A} \exp \left(-2 \rho \sin ^{2}(\pi \eta / \gamma)\langle|x-v t|\rangle\right),
$$

where the angular brackets represent an average of $v$ over $P(v)$ and $\mathcal{A}$ is an $\eta$ and $\gamma$ dependent amplitude [2] related to the vacuum expectation value of $e^{i \eta \Phi}$. Remarkably, Eq. (4) is precisely the result obtained by AT for the integrable sine-Gordon model for generic values of $\gamma$ even away from the free-fermion point. So it appears that the dynamics of the integrable model is rather like that of non-interacting particles.

For generic reflecting soliton case in Fig 1b, with the $S$-matrix in Eq. (2) (which we claim is always the experimentally relevant case), we obtained a very different result. We found

$$
\begin{aligned}
& C_{\Phi}(x, t)=\mathcal{A} e^{-\left(\tilde{q}_{r}+\tilde{q}_{l}\right)}\left[U_{0}\left(2 i \tilde{q}_{r} \Theta, 2 i \sqrt{\tilde{q}_{r} \tilde{q}_{l}}\right)+\right. \\
& U_{0}\left(2 i \tilde{q}_{l} \Theta, 2 i \sqrt{\tilde{q}_{r} \tilde{q}_{l}}\right)-i U_{1}\left(2 i \tilde{q}_{r} \Theta, 2 i \sqrt{\tilde{q}_{r} \tilde{q}_{l}}\right) \\
& \left.-i U_{1}\left(2 i \tilde{q}_{l} \Theta, 2 i \sqrt{\tilde{q}_{r} \tilde{q}_{l}}\right)-I_{0}\left(2 \sqrt{\tilde{q}_{r} \tilde{q}_{l}}\right)\right]
\end{aligned}
$$

where $\Theta \equiv \cos (2 \pi \eta / \gamma), \tilde{q}_{r}=\rho \int_{-\infty}^{x / t} d v P(v)(x-v t), \tilde{q}_{l}=$ $\rho \int_{x / t}^{\infty} d v P(v)(x-v t), I_{0}$ is the modified Bessel function, and $U_{0,1}$ are the Lommel functions of two variables [8]. (Note that all $x$ and and $t$ dependence is through $\tilde{q}_{r}$ and $\tilde{q}_{l}$ which can both be written as functions of the scaling variables $\bar{x}=\rho x \equiv x / \xi_{x}$ and $\bar{t}=c \rho \sqrt{\frac{T}{2 \pi \Delta}} t \equiv t / \xi_{t}$.) 
This remarkable expression is qualitatively different in its asymptotic behavior for large $t$ and $x$ from the corresponding result at the free fermion point. One way to see this is to obtain the correlator of the soliton charge density $\rho=(\gamma / 2 \pi) \partial_{x} \Phi, C_{m}(x, t) \equiv\langle\rho(x, t) \rho(0,0)\rangle$, by expanding Eq. (5) to order $\eta^{2}$ and taking two $x$ derivatives. The result is identical in form to the diffusive correlation function obtained for the conserved (spin) density in Ref 5 using the analysis of Refs 9,10 -here, the diffusion constant has value $D_{m}=\frac{c^{2}}{2 \Delta} e^{\Delta / T}$. A more direct indication of diffusive behavior follows from an asymptotic analysis of our result. For instance, for large $t$ and $x \sim \sqrt{t}$, using $\tilde{q}_{r / l} \simeq t / \xi_{t} \pm x / 2 \xi_{x}$ and standard results for the asymptotics of Bessel and Lommel functions [8], we find:

$$
C_{\Phi}(x, t) \simeq \mathcal{A}\left(\frac{1+\Theta}{\rho(1-\Theta)}\right) \frac{e^{-x^{2} /\left(4 D_{\Phi} t\right)}}{\left(4 \pi D_{\Phi} t\right)^{1 / 2}}
$$

where the diffusion constant $D_{\Phi}=c \sqrt{T} / \rho \sqrt{2 \pi \Delta}=$ $\frac{c^{2}}{2 \Delta} e^{\Delta / T}$ is identical to the diffusion constant $D_{m}$ that describes the diffusive behaviour of the soliton charge correlator. This may be interpreted by noting that the field values $\Phi(x, t)$ and $\Phi(0,0)$ are perfectly correlated if the space-time points $(0,0)$ and $(x, t)$ lie in the same domain, and this happens when the domain walls bounding this domain diffuse from the neighbourhood of the origin to the space-time point $(x, t)$; the equality of the two diffusion constants then suggests that such events dominate in the average over all space-time histories. In this context, it is however important to bear the following subtlety in mind: The asymptotic expression Eq. (6) is only valid as long as $|\Theta|<1-x \xi_{t} /\left(2 t \xi_{x}\right)$, and as such cannot be used directly to expand in $\eta$ about $\eta=0$ (which of course corresponds to $\Theta=1$ ). Put another way, the long time limit does not commute with the $\Theta \rightarrow 1$ limit.

We now describe the derivation of Eqs. (4) and (5). We represent $C(x, t)$ as a 'double time' path integral [1], with the $e^{-i \hat{\mathcal{H}} t}$ factor generating trajectories that move forward in time for each particle, and the $e^{i \hat{\mathcal{H}} t}$ producing trajectories that move backward in time. In the classical limit, stationary phase is achieved when the trajectories are time-reversed pairs of classical paths (Fig 1). Each trajectory has a charge label which obeys Eq. (2) at each collision; however as each collision contributes both to the forward and backward trajectories, the net numerical factor is simply +1 . All of this implies [1] that the straight lines $($ rays $) y_{\mu}(t)=y_{\mu}+v_{\mu} t(\mu=1,2, \ldots M$ )in Fig 1 are independently distributed with a uniform distribution in space for the intercepts $y_{\mu}$, and with inverse slope determined by the velocities $v_{\mu}$ which are independently distributed according to the probability density $P(v)$. The charge, $m$, is assigned randomly to each trajectory at some initial time $t=0$ with probability $\rho_{m} / \rho=1 / 2$, but then evolves in time as discussed above (Fig 1). We have assumed a large system size $L$, and will eventually take the limit $L \rightarrow \infty, M \rightarrow \infty$ with total density $\rho=M / L$ fixed and given by Eq. (11). Note that the charge travelling on a ray changes in time when the $S$-matrix is reflecting, and charges on rays are mutually uncorrelated only at $t=0$ - it is the charges $m_{k}$ ( $k=1,2, \ldots M$ labelled starting from the left) travelling on the complicated zig-zag trajectories $x_{k}(t)$ that remain uncorrelated and constant in time. Conversely, when the $S$-matrix is perfectly transmitting at special free-fermion points in the phase diagram, charges on rays remain uncorrelated and constant in time (since the rays and the trajectories coincide in this case). In both cases, the field $\Phi(x, t)$ is given in terms of soliton trajectories by

$$
\Phi(x, t)=\frac{2 \pi}{\gamma} \sum_{k=1} m_{k} \theta\left(x-x_{k}(t)\right) .
$$

To proceed further in the generic reflecting case, we note that the spatial sequence of domains encountered at any given time as we move from $x=-\infty$ to $x=+\infty$ is invariant under the time evolution. Therefore, it is valid to label the sequence of domains from the left by an index $l$ whereby the field $\Phi$ takes value $\Phi_{l}$ independent of $t$ for all $x$ in the $l^{\text {th }}$ domain. In doing so, we resolve the singularity associated with each collision by stipulating that a zero length domain of the appropriate field value continues to exist at the instant of the collision. Now, let space-time points $(x, t)$ and $(0,0)$ lie in the $l_{2}^{\text {th }}$ and $l_{1}^{t h}$ domain respectively - such an assignment is well-defined precisely because the sequence of domains is time-invariant. The difference $\Phi(x, t)-\Phi(0,0)$ is thus equal to $\Phi_{l_{2}}-\Phi_{l_{1}}$. To obtain the latter, it is extremely useful to work at $t=0$ and write

$$
\Phi_{l_{2}}-\Phi_{l_{1}}=\frac{2 \pi}{\gamma} \sum_{i=1}^{s} m_{i},
$$

where $m_{1 \ldots s}$ are the charges of the $s=\left|l_{2}-l_{1}\right|$ domainwalls (solitons or antisolitions) encountered when going from domain $l_{2}$ to domain $l_{1}$ at $t=0$. The advantage of this procedure is clear: The $m_{1 \ldots\left|l_{2}-1_{1}\right|}$ are uncorrelated independently random variables that take values \pm 1 with equal probability, and as such, may be averaged over independently of one another for fixed $s$.

The number $s$ is of course a complicated function of $x, t$ and all the initial positions $y_{\mu}$ and velocities $v_{\mu}$ of all the rays. However, since the $t=0$ soliton charge on a ray is not correlated with the $y_{\mu}$ and $v_{\mu}$, we may perform the average over these $s$ soliton charges first for a given realization of the $y_{\mu}$ and $v_{\mu}$, and then average the resulting expression over all the $y_{\mu}$ and $v_{\mu}$. The result of this charge averaging is $\left\langle e^{i \eta \Phi(x, t)} e^{-i \eta \Phi(0,0)}\right\rangle_{\text {charge }}=$ $\mathcal{A} \Theta^{s}$.

Next, we note that $s$ may be obtained by moving from the spacetime point $(0,0)$ to point $(x, t)$ along the straight line joining the two, and keeping track of the numbers $k_{r}$ and $k_{l}$ of rays that cross this straight line from the 
right and left respectively. After every such right intersection, we move one domain to the right, while every left intersection results in moving back one domain to the left-thus, $s$ is precisely equal to $\left|k_{r}-k_{l}\right|$. Since initial positions and velocities of the rays are independent random variables, the probability of having $k_{r}$ right intersections and $k_{l}$ left intersections may be calculated straightforwardly by elementary means in terms of $q_{r}$, the probability that the straight line connecting point $(0,0)$ to point $(x, t)$ has a right intersection with a given ray, and $q_{l}$, the corresponding probability for left intersection. This, in conjunction with the result for charge averaging above, then allows us to write the following combinatorial expression

$$
\begin{aligned}
& C_{\Phi}(x, t)=\mathcal{A} \lim _{M \rightarrow \infty} \\
& \sum_{k_{r}, k_{l}} \frac{M ! q_{r}^{k_{r}} q_{l}^{k_{l}}\left(1-q_{r}-q_{l}\right)^{M-k_{r}-k_{l}}}{k_{r} ! k_{l} !\left(M-k_{r}-k_{l}\right) !} \Theta^{\left|k_{r}-k_{l}\right|},
\end{aligned}
$$

where the primed sum is over all $k_{r}$ and $k_{l}$ ranging from 0 to $M$ with the constraint that $k_{r}+k_{l} \leq M, q_{l, r}=\tilde{q}_{l, r} / M$, and we have assumed without loss of generality that $x$ and $t$ are positive.

Before we proceed further, we note that the perfectly transmitting case is considerably simpler: Since the trajectories coincide with rays, and charges on rays are invariant in time and uncorrelated, $\Phi(x, t)-\Phi(0,0)$ can be obtained by moving along the dotted line connecting $(0,0)$ and $(x, t)$ and counting the total number $k_{r}+k_{l}$ of rays intersecting this line (this is the number of uncorrelated random charges we cross in going from $(0,0)$ to $(x, t))$ and multiplying by $2 \pi / \gamma$. The rest of the argument above is unchanged and we obtain a simpler combinatorial expression with the $\left|k_{r}-k_{l}\right|$ in the exponent of the cosine in Eq. (9) replaced by $k_{r}+k_{l}$. Taking the thermodynamic limit using the trinomial formula gives the result Eq. (4).

Returning to the reflecting case, further progress relies crucially on a device suggested to us by D. Dhar. The artifice involved consists of viewing the sum of a subset of terms with fixed $k_{r}-k_{l}=n$ as the coefficient of $z^{n}$ in the Laurent expansion of some function of a complex variable $z$. For $n \geq 0$, this is achieved by identifying the corresponding partial sum with the coefficient of $z^{n}$ in the trinomial expansion of $f_{M}(\Theta z) \equiv$ $\left(1-q_{r}-q_{l}+\Theta z q_{r}+q_{l} / \Theta z\right)^{M}$. Similarly, for $n<0$, the identification is with the coefficient of $z^{n}$ in the trinomial expansion of $f_{M}(z / \Theta)$. The advantage of this approach is now clear: Each Laurent coefficient can be written as a contour integral around a contour enclosing the origin, and the two sums over $n$ for either sign of $n$ are simply geometric series which can be evaluated in closed form. Moreover, the thermodynamic limit can be taken explicitly for both sums upon choosing in each case a contour along which the geometric series converges. A convenient rescaling of integration variables then gives:

$$
\begin{aligned}
C_{\Phi}(x, t) & =\mathcal{A} \frac{e^{-\left(\tilde{q}_{r}+\tilde{q}_{l}\right)}}{2 \pi i}\left(\oint_{\mathcal{C}_{a}} \frac{d w}{w-\Theta\left(\frac{\tilde{q}_{r}}{\tilde{q}_{l}}\right)^{\frac{1}{2}}} e^{\sqrt{\tilde{q}_{r} \tilde{q}_{l}}\left(w+w^{-1}\right)}\right. \\
+ & \left.\oint_{\mathcal{C}_{b}} \frac{d w}{w-\frac{1}{\Theta}\left(\frac{\tilde{q}_{r}}{\tilde{q}_{l}}\right)^{\frac{1}{2}}} e^{\sqrt{\tilde{q}_{r} \tilde{q}_{l}}\left(w+w^{-1}\right)}\right)
\end{aligned}
$$

Here $\mathcal{C}_{a}$ is an anti-clockwise contour enclosing the origin with $|w|>|\Theta| \sqrt{\tilde{q}_{r} / \tilde{q}_{l}}$ and $\mathcal{C}_{b}$ goes clockwise around the origin with $|w|<\sqrt{\tilde{q}_{r} / \tilde{q}_{l}} /|\Theta|$. We now expand the exponentials in a Laurent series and integrate each term to finally arrive at Eq. (5) upon resumming the resulting series.

The main issue raised by our results is that of the origin of the distinction between the semiclassical and formfactor results. Both methods make subtle unproven assumptions about orders of limits: the commuting of the $t \rightarrow \infty$ limit with either the semiclassical (in which the low momentum limit is taken in obtaining Eq. (2)) or the form factor expansions. It could be that such an assumption is invalid in one of the methods, and only the other result is generically valid in the long-time limit. However, we suspect that the distinction reflects a fundamental physical difference between generic and integrable systems, and the two results apply in their respective cases.

After this work was completed, we became aware of independent work by Rapp and Zarand 11] who find that diffusive behavior appears in the correlators of the onedimensional $Q$-state quantum potts model for $Q>2$. Remarkably, the correlation functions in the two cases differ only in the choice of the parameter $\Theta$ which takes the value $\Theta=-1 /(Q-1)$ in the Potts case.

We would like to acknowledge A. Tsvelik for useful discussions regarding the results obtained in $\operatorname{Ref} 2$, and thank G. Zarand for useful discussions regarding the the results of Ref 11 . One of us (KD) would like to thank A. Dighe and R. Raghunathan for useful discussions, and D. Dhar for insightful discussions and suggesting the trick used in arriving at an integral representation of Eq. (9). S.S. was supported by NSF Grant DMR-0537077.

[1] S. Sachdev and A. P. Young, Phys. Rev. Lett. 78, 2220 (1997).

[2] B. L. Altshuler and A. M. Tsvelik, cond-mat/0505367 unpublished.

[3] S. Fujimoto, J. Phys. Soc. Jpn. 68, 2810 (1999).

[4] R. M. Konik, Phys. Rev. B 68, 104435 (2003).

[5] K. Damle and S. Sachdev, Phys. Rev. B 57, 8307 (1998).

[6] S. Mukerjee, V. Oganesyan, and D. A. Huse, cond-mat/0503177 unpublished.

[7] F. H. L. Essler and R. M. Konik, From Fields to Strings: Circumnavigating Theoretical Physics, M. Shifman, A. Vainshtein, and J. Wheather (eds), World Scientific (2005); cond-mat/0412421. 
[8] G. N. Watson, A Treatise on the Theory of Bessel Functions, Cambridge University Press (1944).

[9] D. W. Jepsen, J. Math. Phys. 6, 405 (1965).

[10] J. L. Lebowitz and J. K. Percus, Phys. Rev 155, 122
(1967).

[11] A. Rapp and G. Zarand, cond-mat/0507390 